\title{
The Common miRNA Signatures Associated with Mitochondrial Dysfunction in Different Muscular Dystrophies
}

Evrim Aksu-Menges, ${ }^{*}$ Yeliz Z. Akkaya-Ulum, ${ }^{*}$ Didem Dayangac-Erden, ${ }^{*}$ Banu Balci-Peynircioglu, ${ }^{*}$ Ayse Yuzbasioglu, * Haluk Topaloglu, ${ }^{\dagger}$ Beril Talim, ${ }^{\ddagger}$ and Burcu Balci-Hayta*

From the Department of Medical Biology* and the Division of Child Neurology ${ }^{\dagger}$ and the Pathology Unit, ${ }^{\ddagger}$ Department of Pediatrics, Hacettepe University Faculty of Medicine, Ankara, Turkey

Accepted for publication June 22, 2020.

Address correspondence to Burcu Balci-Hayta, Ph.D., Hacettepe University, Faculty of Medicine, Rectorate Building, $7^{\text {th }}$ floor, Department of Medical Biology, 06100, Sihhiye, Ankara, Turkey. E-mail: burcub@hacettepe.edu.tr.

\begin{abstract}
Secondary mitochondrial damage in skeletal muscles is a common feature of different neuromuscular disorders, which fall outside the mitochondrial cytopathies. The common cause of mitochondrial dysfunction and structural changes in skeletal muscle tissue remains to be discovered. Although they are associated with different clinical, genetic, and pathologic backgrounds, the pathomechanisms underlying neuromuscular disorders might be attributed to the complex interaction and cross talk between mitochondria and the associated miRNAs. This study aimed to identify the common miRNA signatures that are associated with mitochondrial damage in different muscular dystrophies (MDs; Duchenne muscular dystrophy, megaconial congenital muscular dystrophy, Ullich congenital muscular dystrophy, and $\alpha$-dystroglycanopathy). The miRNome profiles of skeletal muscle biopsies acquired from four different MD groups and control individuals were analyzed by miRNA microarray. We identified 17 common up-regulated miRNAs in all of the tested MD groups. A specific bioinformatics approach identified 10 of these miRNAs to be specifically related to the mitochondrial pathways. Six miRNAs, miR134-5p, miR-199a-5p, miR-382-5p, miR-409-3p, miR-497-5p, and miR-708-5p, were associated with the top four mitochondrial pathways and were thus selected as priority candidates for further validation by quantitative real-time PCR analysis. We demonstrate, for the first time, common up-regulated miRNAs that are associated with mitochondrial damage in different MD groups, therefore contributing to the pathophysiology. Our findings may open a new gate toward therapeutics. (Am J Pathol 2020, 190: 2136-2145; https://doi.org/10.1016/j.ajpath.2020.06.011)
\end{abstract}

Neuromuscular disorders (NMDs) are a heterogeneous and large group of diseases that mainly affect the skeletal muscles, motor nerves, and/or neuromuscular junctions, and are characterized by impaired muscle functioning and progressive physical disability. Although some of the NMDs belong to the group of rare genetic disorders, $>780$ different types of NMDs and 417 responsible genes have been identified to date. ${ }^{1,2}$ A subset of NMDs, muscular dystrophies (MDs), are inherited diseases that cause weakness and degeneration of the skeletal muscles, and are usually caused by mutations in the genes that are responsible for the structure and function of muscles. ${ }^{3}$ Although the causative genes and pathologic basis responsible for these MDs vary, mitochondrial dysfunction in skeletal muscle, detected mostly by using ultra-high-resolution techniques, is observed as a common feature among most of them. Ultrastructural analysis of mitochondria by confocal microscopy or transmission electron microscopy showed abnormal mitochondrial morphology in patients and disease models. Moreover, in vitro functional assays demonstrated altered mitochondrial membrane potential and energy metabolism and increased reactive oxygen species production in the pathogenesis of these disorders. ${ }^{4-11}$ Recent

\footnotetext{
Suppported by Hacettepe University Scientific Research Projects Coordination Unit Project number TSA-2017-14413.

Disclosures: None declared.

Portions of these data were presented at the $43^{\text {rd }}$ Federation of European Biochemical Societies (FEBS) Congress, on July 7-12, 2018, Prague, Czechia.
} 
studies have shown that mitochondrial dysfunction in skeletal muscle, which is caused by unknown factors present in cellular microenvironment, plays a crucial role in early stages of muscle degeneration. ${ }^{12-16}$ Thus, identifying the potential role of miRNAs that are associated with the mitochondrial damage pathways is a prevalent research topic.

miRNAs are endogenous small noncoding RNA molecules that are involved in the post-transcriptional regulation of gene expression by the RNA interference mechanism. ${ }^{17}$ It is known that miRNAs can be encoded by both nuclear and mitochondrial genomes, and they have been found to play important roles in regulating mitochondrial function, depending on the metabolic demands. ${ }^{18,19}$ Besides being relevant to mitochondrial function, cellular expression levels of miRNAs could also be used as potential diagnostic, prognostic, and therapeutic targeted biomarkers. ${ }^{20}$ In the light of this information, it is suggested that miRNAs associated with mitochondrial function might be one of the most important factors related to mitochondrial damage observed in the pathogenesis of various NMDs and might act as potential tissue-specific biomarkers that could lead to the development of alternative and common therapeutic options in this large group of disorders.

There have been several reports focusing on muscle-specific miRNA profiling of a single NMD. For instance, Zaharieva et $\mathrm{al}^{21}$ (2013) showed that muscle-specific miRNAs, miR-1, miR-133, and miR-206, were highly elevated in the serum of Duchenne muscular dystrophy (DMD) patients; however, the expression of these miRNAs remained unchanged in the serum of Ullrich congenital muscular dystrophy (CMD) patients. In another study, it was shown that the levels of 14 miRNAs, including muscle-specific miRNA, in the serum samples of adult-onset Pompe disease patients, were elevated compared with the control samples. ${ }^{22}$ There are other studies that also revealed the importance of miRNAs in the maintenance of mitochondrial function in skeletal muscle cells. For instance, it has been reported that in murine myoblasts, miR-494 regulates mitochondrial biogenesis by targeting mitochondrial transcription factor A (mtTFA, also known as Tfam) and forkhead box j3 (Foxj3). ${ }^{23}$ In addition, miR-181a was shown to regulate mitophagy by suppressing autophagy regulatory proteins, such as p62 (sequestosome 1); and mitophagy-associated proteins, such as Parkin (Park2) and Parkinson protein 7 (Park7) in murine myoblasts. ${ }^{24}$ In another study, Mohamed et al ${ }^{25}$ (2014) revealed that down-regulation of miR-149 positively regulates mitochondrial function and biogenesis by targeting the poly(ADP-ribose) polymerase-2 (PARP2) gene in myoblast cells. In human skeletal muscle, miR-542-5p has been shown to negatively regulate mitochondrial respiratory chain and membrane potential by down-regulating several genes, including SMAD family member 7 (SMAD7), SMAD specific E3 ubiquitin protein ligase 1 (SMURF1), and protein phosphatase 2 catalytic subunit $\alpha(P P P 2 C A) .{ }^{26}$ However, there is no study to date that discussed the mitochondria-related miRNAs involved in the pathogenesis of MDs that have mitochondrial dysfunction as a common feature.

In this study, we identified, for the first time, the common miRNA signatures associated with mitochondrial damage in different MDs, including DMD, megaconial CMD, Ullrich $\mathrm{CMD}$, and $\alpha$-dystroglycanopathy ( $\alpha \mathrm{DG}$ athy), as all of these MDs are caused by mutations in different genes encoding for proteins with distinct functions.

\section{Materials and Methods}

\section{Patients}

In this study, the experimental protocol was approved by the Hacettepe University Faculty of Medicine Ethical Review

Table 1 The Clinical Diagnosis and Histopathologic Characteristics of the Patients

\begin{tabular}{|c|c|c|c|c|c|c|c|c|c|}
\hline $\begin{array}{l}\text { Case } \\
\text { no. }\end{array}$ & $\begin{array}{l}\text { Age, } \\
\text { years }\end{array}$ & $\begin{array}{l}\text { Clinical } \\
\text { features }\end{array}$ & $\begin{array}{l}\text { Diagnostic histologic } \\
\text { findings }\end{array}$ & $\begin{array}{l}\text { Microscopic mitochondrial } \\
\text { changes }\end{array}$ & Degeneration & Regeneration & Fibrosis & Adiposis & Inflammation \\
\hline D1 & 11 & DMD & Dystrophin negative & - & ++ & ++ & ++ & ++ & ++ \\
\hline D2 & 3 & DMD & Dystrophin negative & - & +++ & +++ & ++ & + & ++ \\
\hline D3 & 5 & DMD & Dystrophin negative & - & +++ & +++ & ++ & + & ++ \\
\hline $\mathrm{A} 1$ & 1.5 & CMD & aDG negative & - & ++ & + & +++ & ++ & ++ \\
\hline A2 & 2.5 & CMD & $\alpha \mathrm{DG}$ negative & - & +++ & +++ & ++ & + & ++ \\
\hline U2 & 6 & MD & Collagen VI negative & - & + & + & +++ & +++ & + \\
\hline U3 & 2.5 & CMD & Collagen VI negative & - & + & + & + & + & + \\
\hline M1 & 14 & MD & Mega mitochondria & Mega mitochondria & + & + & + & + & - \\
\hline M2 & 2 & CMD & Mega mitochondria & Mega mitochondria & + & + & + & + & - \\
\hline M3 & 6 & CMD & Mega mitochondria & $\begin{array}{l}\text { Mega mitochondria, } \\
\text { few COX-negative fibers }\end{array}$ & + & + & + & ++ & - \\
\hline
\end{tabular}

D1 to D3, DMD; A1 to A3, $\alpha$-dystroglycanopathy; U1 to U3, Ullrich CMD; M1 to M3, megaconial CMD; and C1 to C3, control.

- , Absent; + , mild; ++ , moderate; +++ , severe; $\alpha$ DG, $\alpha$-dystroglycan; CMD, congenital MD; COX, cytochrome C oxidase; DMD, Duchenne MD; MD, muscular dystrophy. 
Board (GO 16/358-25). Skeletal muscle biopsies from 3 control subjects (age range, 1.5 to 16 years; mean age, 9.17 years) and 12 patients with clinicopathologic and/or molecular diagnosis of four different muscular dystrophies (DMD, megaconial CMD, Ullrich CMD, and aDGpathy; 3 patients from each group) (age range, 2 to 14 years; mean age, 5 years) were included in this study. Muscle biopsies with no diagnostic pathology, as indicated by normal histomorphology, were used as controls. Genetic analysis for $\alpha$ DGpathy cases has not been performed at the time of study. The biopsies were acquired from the vastus lateralis muscle at the time of diagnosis, after informed consent. Specimens were rapidly frozen in isopentane cooled in liquid nitrogen and stored at $-80^{\circ} \mathrm{C}$ until further use. Standard histologic and histochemical techniques were applied to the cryostat tissue sections, including hematoxylin and eosin, modified Gomori trichrome, periodic acid-Schiff, oil red O, NAD dehydrogenase-tetrazolium
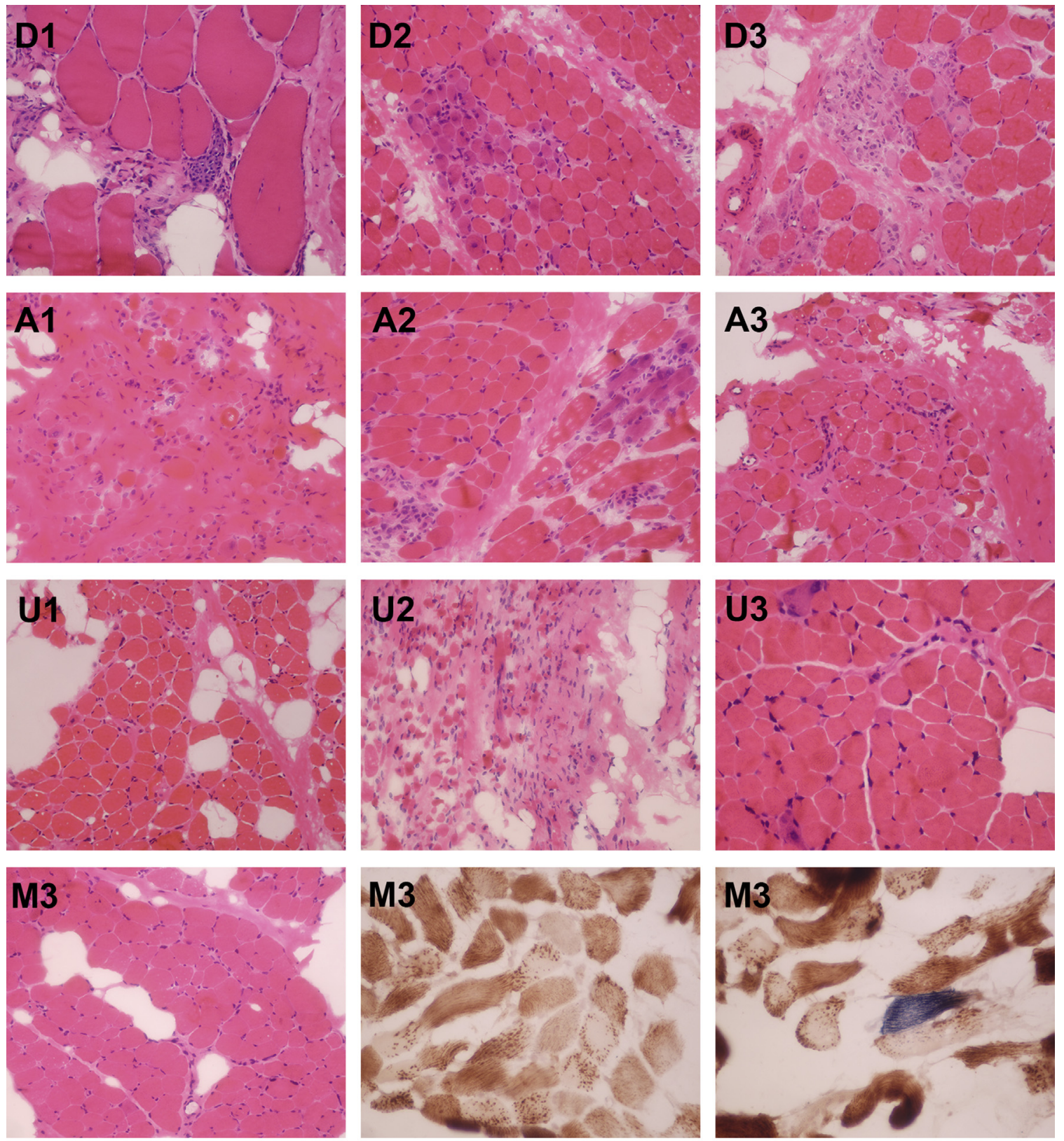

Figure 1 Muscle biopsies showing a spectrum of dystrophic changes (hematoxylin and eosin stain). Duchenne muscular dystrophy biopsies with moderate fibrosis, adiposis, and inflammation (D1 and D3), and many regenerating and degenerating fibers (D2 and D3, respectively). $\alpha$-Dystroglycanopathy biopsies with prominent fiber damage and regeneration (A2) and moderate (A3) to severe (A1) fibrosis. Ullrich congenital muscular dystrophy (CMD) biopsies with moderate (U1) to severe (U2) fibrosis and adiposis and rare regenerating fibers (U3). Megaconial CMD biopsy (M3) with mild fibrosis and moderate adiposis. Cytochrome $\mathrm{C}$ oxidase-succinate dehydrogenase (COX/brown-SDH/blue) stained sections: Mitochondria are strikingly enlarged and localized toward the sarcolemma; in some fibers, central area is devoid of mitochondria. One COX-negative fiber is also seen (blue). Original magnification: $\times 40$ (COX-SDH stain); $\times 20$ (all others). 
reductase, succinate dehydrogenase, cytochrome $\mathrm{C}$ oxidase, and ATPase. Immunohistochemical and/or immunofluorescence analyses were performed as appropriate, using the antibodies against dystrophins, sarcoglycans, laminin $\alpha 2$ (merosin), collagen VI, and $\alpha$-dystroglycan $(\alpha \mathrm{DG})$. Archive sections were evaluated for histologic dystrophic and mitochondrial changes. Degeneration, regeneration, fibrosis, adiposis, and inflammation were scored as absent, mild, moderate, or severe.

\section{RNA Isolation from Fresh-Frozen Skeletal Muscle Biopsies}

Total RNAs, including the miRNAs, were isolated from the skeletal muscle biopsies (approximately $30 \mathrm{mg}$ ) of 12 patients representing four different MDs and 3 control subjects using the miRCURY RNA Isolation Kit-Cell \& Plant (Qiagen, Valencia, $\mathrm{CA}$ ), according to the manufacturer's instructions. RNA integrity and concentration were assessed using the agarose gel electrophoresis and NanoDrop ND-1000 spectrophotometer (Thermo Scientific, Waltham, MA), respectively.

\section{miRNA Microarray}

The miRNome profiles of all the 15 samples were analyzed by GeneChip miRNA 4.0 Array (Affymetrix by Thermo Fisher Scientific, Santa Clara, CA), containing probes of 2578 mature human miRNAs, according to the manufacturer's instructions. Total RNA (900 ng) was used as the starting material for microarray analysis. After miRNAs were extended with a $3^{\prime}$-poly(A) tail using poly(A) polymerase, a biotin tag was ligated to the poly(A) tail for fluorescent dye staining in the later steps. Hybridization with biotinylated RNAs was performed in a hybridization oven at $48^{\circ} \mathrm{C}$ for overnight. Biotin detection was performed using the streptavidin-horseradish peroxidase, and the hybridization images were digitized using an Affymetrix GeneChip Scanner $30007 \mathrm{G}$ and the Expression Console Software version 1.2 (Affymetrix by Thermo Fisher Scientific). As a quality control measure of the miRNA microarray experiment, signal intensities (presence/absence values) and signal histograms of all of the chips were examined (Supplemental Figure S1). To identify the differentially expressed miRNAs in the patients, raw data were analyzed by using the Multi-Experiment Viewer $(\mathrm{MeV}$ ) version 4.9.0 (The Institute for Genomic Research, Rockville, MD) and Affymetrix Transcriptome Analysis Console Version 2. Differentially expressed miRNAs that were found to be statistically significant by using both the programs (with parameters, fold change $\geq 2.0$ and false discovery rate $=0$ for Multi-Experiment Viewer $(\mathrm{MeV})$-significance analysis of microarrays analysis; and $P<0.05$ for Transcriptome Analysis Console-analysis of variance analysis) were identified as the potential miRNA candidates.

\section{Computational Prediction of miRNA Targets and Gene Enrichment Analysis}

The potential mitochondrial target genes of candidate miRNAs were identified by using miRWalk 2.0 database. By adopting a specific bioinformatics approach, the list of genes encoding for the mitochondrial proteome (both nuclear and mitochondrial genes) were obtained from Human MitoCarta2.0. The mitochondrial genes were sorted out

Table 2 The List of Differentially Up-Regulated miRNAs with a Fold Change $\geq 2$ and $P<0.05$ in MD Groups Compared with Control Subjects

\begin{tabular}{lcccc}
\hline \multirow{2}{*}{$\begin{array}{l}\text { Differentially expressed } \\
\text { miRNAs in MD groups }\end{array}$} & \multicolumn{2}{c}{ Multi-Experiment Viewer } & & \multicolumn{2}{c}{ Transcriptome Analysis Console } \\
\cline { 2 - 5 } & Fold change & FDR, $\%$ & Fold change & Analysis of variance $P$ value \\
\hline hsa-miR-146b-5p & 42.36 & 0.00 & 36.09 & $1.22 \times 10^{-7 * * *}$ \\
hsa-miR-379-5p & 5.24 & 0.00 & 4.7 & $0.000001^{* *}$ \\
hsa-miR-199a-5p & 5.61 & 0.00 & 5.69 & $0.024402^{*}$ \\
hsa-miR-382-5p & 6.95 & 0.00 & 6.4 & $0.000018^{* * *}$ \\
hsa-miR-432-5p & 9.78 & 0.00 & 8.77 & $0.000028^{* * *}$ \\
hsa-miR-411-5p & 11.53 & 0.00 & 9.56 & $0.000057^{* * *}$ \\
hsa-miR-409-3p & 3.59 & 0.00 & 3.69 & $0.000166^{* * *}$ \\
hsa-miR-24-2-5p & 4.11 & 0.00 & 4.04 & $0.000199^{* * *}$ \\
hsa-miR-134-5p & 8.46 & 0.00 & 9.57 & $0.000348^{* * *}$ \\
hsa-miR-708-5p & 13.54 & 0.00 & 5.14 & $0.000445^{* * *}$ \\
hsa-miR-497-5p & 3.33 & 0.00 & 3.43 & $0.000457^{* * *}$ \\
hsa-miR-214-3p & 3.56 & 0.00 & 5.46 & $0.000589^{* * *}$ \\
hsa-miR-21-5p & 9.63 & 0.00 & 3.24 & $0.001028^{* *}$ \\
hsa-miR-155-5p & 3.43 & 0.00 & 5.09 & $0.000942^{* * *}$ \\
hsa-miR-487b-3p & 5.78 & 0.00 & 4.27 & $0.001245^{* *}$ \\
hsa-miR-127-3p & 5.28 & 0.00 & 5.17 & $0.001628^{* *}$ \\
hsa-miR-424-3p & 6.99 & 0.00 & $0.002205^{* *}$
\end{tabular}

Statistical analysis was performed using significance analysis of microarrays.

${ }^{*} P<0.05,{ }^{* *} P<0.01$, and ${ }^{* * *} P<0.001$.

FDR, false discovery rate; MD, muscular dystrophy. 


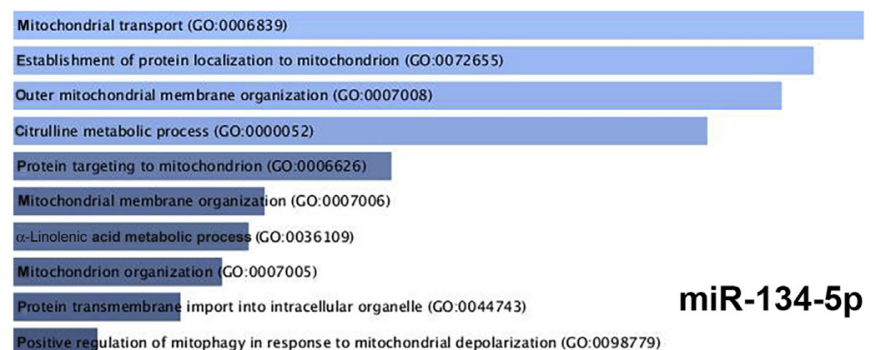

Positive regulation of mitophagy in response to mitochondrial depolarization (GO:0098779)

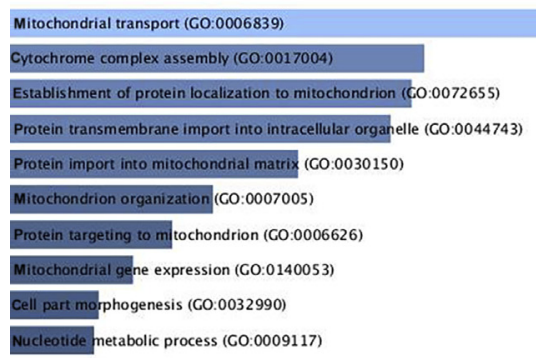

miR-382-5p

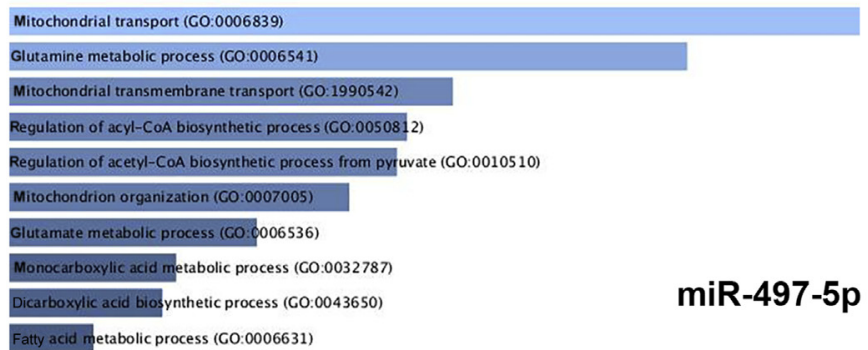

Mitochondrial translational elongation (G0:0070125)

Trans lational elongation (GO:0006414)

Mitochondrial translation (GO:0032543)

Mitochondrial translational termination (G0:0070126)

Translational temination (60:0006415)

Mitochondrial transport (60:0006839)

Respiratory electron transport chain (GO:0022904)

Mitochondrial respiratory chain complex assembly (GO:0033108)

Mitochondrion organization (GO:0007005)

Glyoxylate metabolic process (GO:0046487)
miR-199a-5p

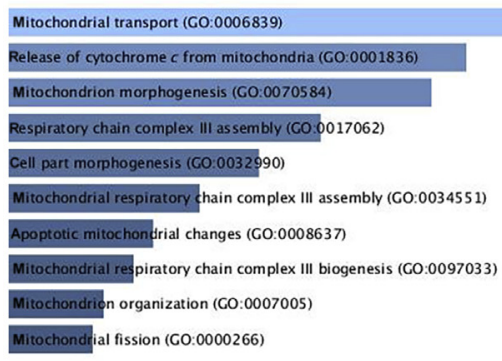

miR-409-3p

Establishment of protein localization to mitochondrion (CO:0072655)

Mitochondrial transport (CO:0006839)

Regulation of acyl-COA biosynthetic process (CO.0050812)

Regulation of acetyl-COA biosynthetic process from pyruvate (CO:0010510)

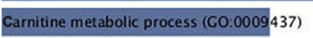

Protein targeting to mitochondrion (CO:0006626)

Mitochondrial calcium uptake (CO:0036444)

Mitochondrion organization (CO:0007005)

Coenzyme metabolic process (CO:0006732)

Glutamine metabolic process (CO:0006541)
miR-708-5p

Figure 2 Gene Ontology (G0) term enrichment analysis of the mitochondrial target genes of miR-134-5p, miR-199a-5p, miR-382-5p, miR-409-3p, miR497-5p, and miR-708-5p within the Biological Process category. Bar graphs are sorted by $P$ value $(P<0.05)$. The longer bars and lighter colored bars mean that the term/gene set is more significant.

from the target gene list by Venny 2.1.0 tool for each miRNA. Gene Ontology enrichment analysis was conducted using all the mitochondrial target genes of each candidate miRNA based on the biological processes.

\section{Expression Validation of Candidate miRNAs Using Quantitative Real-Time PCR}

To validate the difference in the expression levels of candidate miRNAs in NMD groups, quantitative real-time PCR analyses were performed. Reverse transcription was performed by using the miRNA-specific stem-loop RT primers (TaqMan MicroRNA Assays; Thermo Fisher Scientific) and the TaqMan MicroRNA Reverse Transcription Kit (Thermo Fisher Scientific). Subsequently, quantitative real-time PCR was performed with TaqMan probes (TaqMan MicroRNA Assays; Thermo Fisher Scientific) and TaqMan Universal PCR Master Mix, and no AmpErase UNG (Thermo Fisher Scientific). All reactions were performed with Bio-Rad IQ5 Real-Time PCR Detection Systems (Bio-Rad, Hercules, CA). The $2_{\mathrm{T}}^{-\Delta \Delta \mathrm{C}}$ method was used to analyze the relative changes in the expression levels of candidate miRNAs. Each sample was tested in triplicate, and all quantifications were normalized using U6 small nuclear RNA.

\section{Statistical Analysis}

For the miRNA microarray analysis, the significance analysis of microarrays and analysis of variance test were applied to identify the differentially expressed miRNAs in Multi-Experiment Viewer (MeV) and Transcriptome Analysis Console programs, respectively. For the quantitative real-time PCR experiments, fold changes in the expression level of miRNAs in MD groups were evaluated by $U$-test with $P<0.05$ being considered as significant. Statistical analyses were performed using the GraphPad Prism 5.0 software (GraphPad Software, Inc., San Diego, CA).

\section{Results}

\section{Histologic Evaluation}

Muscle biopsies from a total of 12 patients from DMD, megaconial CMD, Ullrich CMD, and $\alpha$ DGpathy and 3 control subjects were included in the study. Age and clinical and histopathologic features are given in Table 1. Dystrophic findings were more severe in DMD and $\alpha$ DGpathy cases (Figure 1). As expected, microscopical mitochondrial changes were present only in megaconial $\mathrm{CMD}$, in the form of mega 

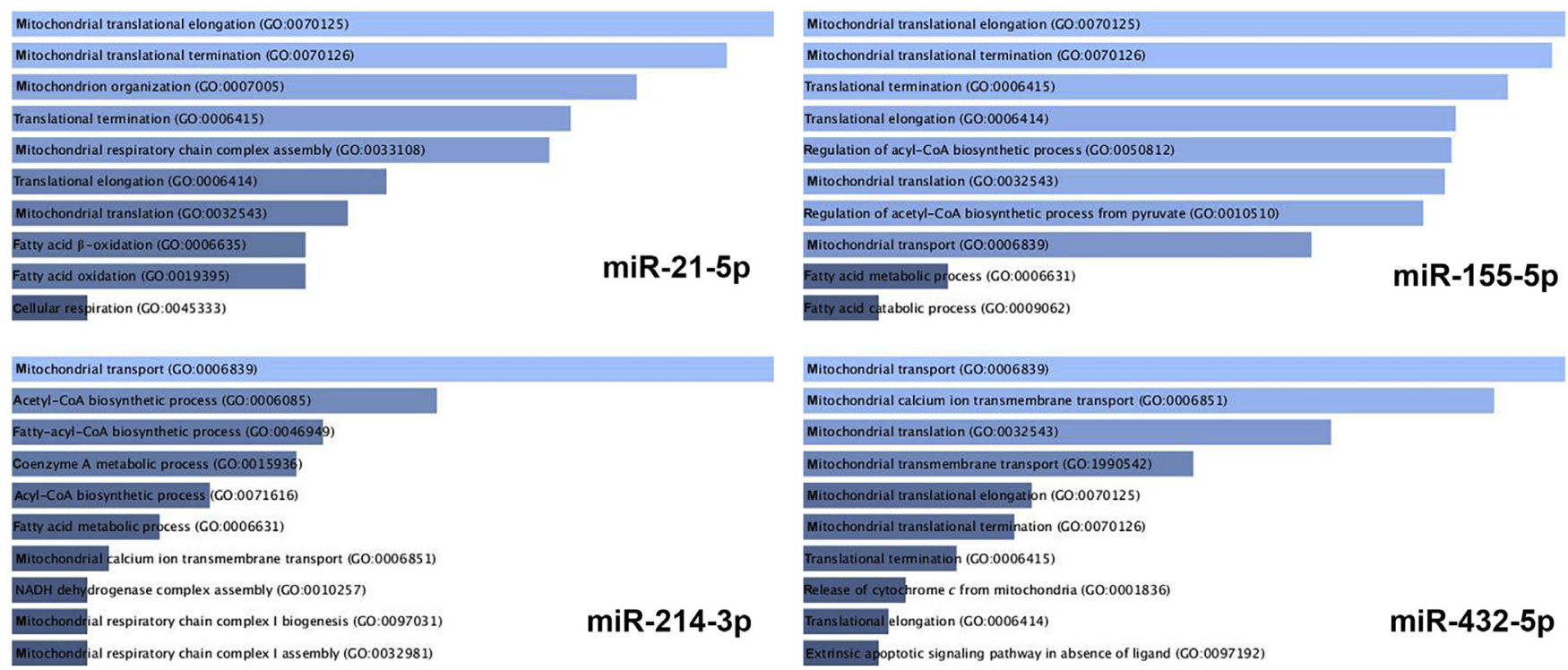

Figure 3 Gene Ontology (G0) term enrichment analysis of the mitochondrial target genes of miR-21-5p, miR-155-5p, miR-214-3p, and miR-432-5p within the Biological Process category. Bar graphs are sorted by $P$ value $(P<0.05)$. The longer bars and lighter colored bars mean that the term/gene set is more significant.

mitochondria showing typical peripheral distribution within the muscle fibers. One megaconial CMD biopsy also showed few cytochrome $\mathrm{C}$ oxidase-negative fibers (Figure 1).

\section{miRNA Microarray Analysis Reveals 17 Differentially Expressed miRNAs in MD Groups}

To identify the differentially expressed miRNAs that are associated with the mitochondrial damage in four MD groups, a miRNA microarray analysis was performed. The miRNA quality control tool (Affymetrix) was used for the evaluation of the quality of miRNA microarrays. Thereafter, robust multichip average (RMA) and detection above background (DABG) algorithms of the Expression Console Software version 1.2 (Affymetrix) were used for background correction, data normalization, and the computation of the probe-set level expression (Supplemental Figure S1). When compared with the control subjects, 17 miRNAs were found to be up-regulated in MDs with a fold change threshold $\geq 2.0$, false discovery rate $=0$, and $P<0.05$, according to both the Multi-Experiment Viewer $(\mathrm{MeV})$ and Transcriptome Analysis Console 2.0 programs (Table 2). The data discussed in this publication have been deposited in the ArrayExpress database at The European Molecular Biology Laboratory - The European Bioinformatics Institute (EMBL-EBI; http://www.ebi.ac.uk/arrayexpress; accession number E-MTAB-9030).

\section{Target Genes of 10 Candidate miRNAs Are Associated with Mitochondrial Pathways}

The potential target genes of selected miRNAs were identified by using miRWalk 2.0, a comprehensive database of predicted as well as the experimentally validated miRNA targets. Thereafter, the mitochondrial genes were sorted from the available gene list, and the putative mitochondrial target genes of candidate miRNAs were subjected to Gene Ontology enrichment analysis in terms of the biological process domain. From this analysis, it was revealed that 10 of 17 selected miRNAs, miR-21-5p, miR-134-5p, miR-155-5p, miR-199a-5p, miR-214-3p, miR-382-5p, miR-409-3p, miR-432-5p, miR-497-5p, and miR-708-5p, which showed increased expression in MD groups by microarray analysis, were associated with mitochondrial pathways (Figures 2 and 3). The top four significantly enriched Gene Ontology pathways were mainly related to mitochondrial transport, mitochondrial organization, mitochondrial electron transport, and mitochondrial transmembrane transport. The complete mitochondrial target gene list of these 10 differentially expressed miRNAs in different MD groups is presented in Supplemental Table S1.

Validation of the Expression Levels of Selected miRNAs in Patient Samples by Quantitative Real-Time PCR Analysis

Of these 10 differentially expressed miRNAs, 6 miRNAs, miR-134-5p, miR-199a-5p, miR-382-5p, miR-409-3p, miR497-5p, and miR-708-5p, that were associated with top four mitochondrial pathways were identified as the priority candidates. According to the miRNA microarray analysis, miR-134-5p and miR-409-3p were markedly elevated in DMD group. Among up-regulated miRNAs, the highest increase of miR-199a-5p, miR-497-5p, and miR-708-5p was detected in Ullrich CMD patients. Also, it was determined that the group with the highest increase of miR-382-5p was $\alpha$ DGpathy. However, there is no relationship between histopathologic severity and the expression of the six miRNAs. Experimental validation of the expression of these six 

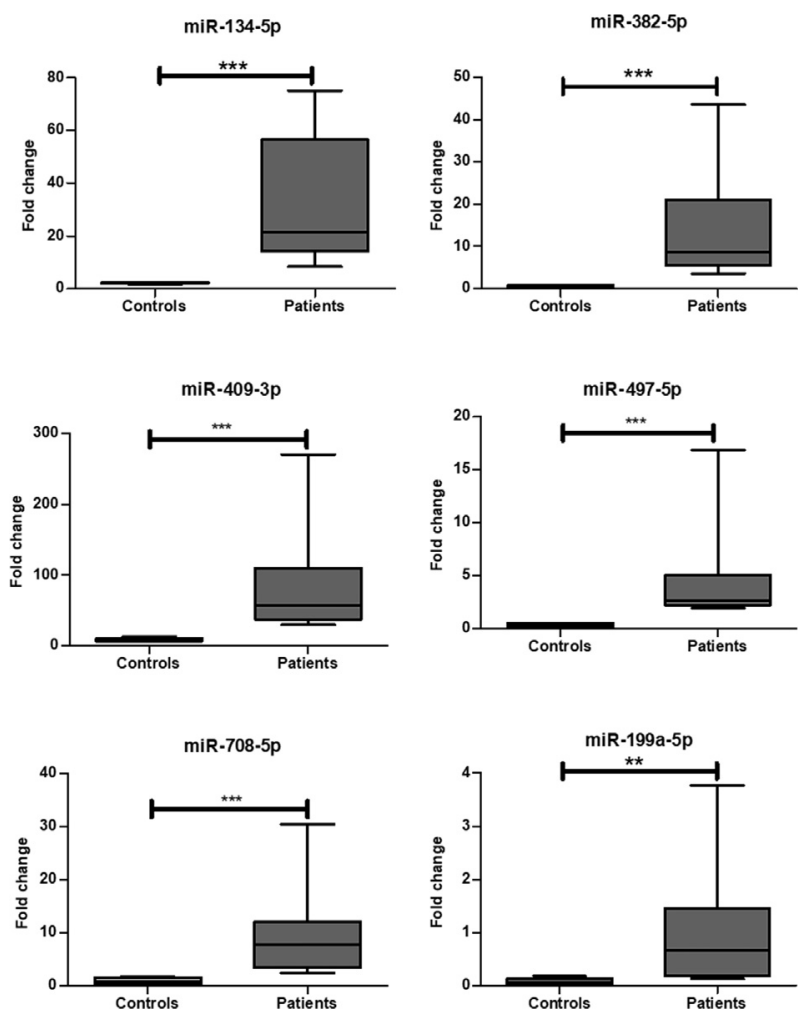

Figure 4 Quantitative real-time PCR validation of six differentially expressed miRNAs associated with top four enriched mitochondrial pathways. The relative expression of miRNAs was normalized to U6 small nuclear RNA, by using the $2_{T}^{-\Delta \Delta C}$ method. Statistical significance was determined using the $U$-test. The median value is represented by a horizontal line inside the box, where boxes represent upper and lower quartiles and whiskers show maximum and minimum values. ${ }^{* *} P<0.01,{ }^{* * *} P<0.001$.

miRNAs by quantitative real-time PCR analysis revealed that all of them were significantly up-regulated in different MDs when compared with control subjects (Figure 4). A similar increase pattern was observed in all MD groups. The main results of our study are summarized in the form of a flowchart representation in Figure 5. These six miRNAs might have potential role in mitochondrial damage pathways and thus were chosen as candidates for the future experiments.

\section{Discussion}

There are about 7000 rare diseases that affect approximately 400 million people worldwide. So far, there is no approved treatment for about 95\% of them (RARE Facts-Global Genes, https://globalgenes.org/rare-facts, last accessed December 12, 2019). These rare diseases are now being increasingly characterized by common pathogenic mechanisms (commonalities) instead of being studied as single disorder, and many different diagnostic/therapeutic approaches are being developed, which would be applicable in case of many of these disorders. ${ }^{27}$ NMDs are a heterogeneous group of rare disorders that causes progressive muscle weakness and affects the patients' quality of life severely. Even if the number of individuals affected by a specific type of NMD is low, all together the occurrence of different types of NMDs affect a large population worldwide, which makes them a challenging health issue. ${ }^{2}$ They have a great impact on the patients' quality of life, which underlies the importance of developing new diagnostic and therapeutic approaches to treat them. ${ }^{28}$ Intensive studies have been performed in this regard; however, no effective common treatment option has been found to date. However, therapeutic strategies often implement a single-gene-single-target approach, and thus preclinical studies have paved the way for individualized innovative treatment for such diseases. ${ }^{29,30}$

Secondary mitochondrial dysfunction and dysmorphology in skeletal muscle tissues have been linked to the pathomechanisms of the wide variety of NMDs that are related to different pathogenic mechanisms, such as DMD, limb-girdle muscular dystrophy type 2A, megaconial CMD, Ullrich CMD, Bethlem myopathy, centronuclear myopathies,

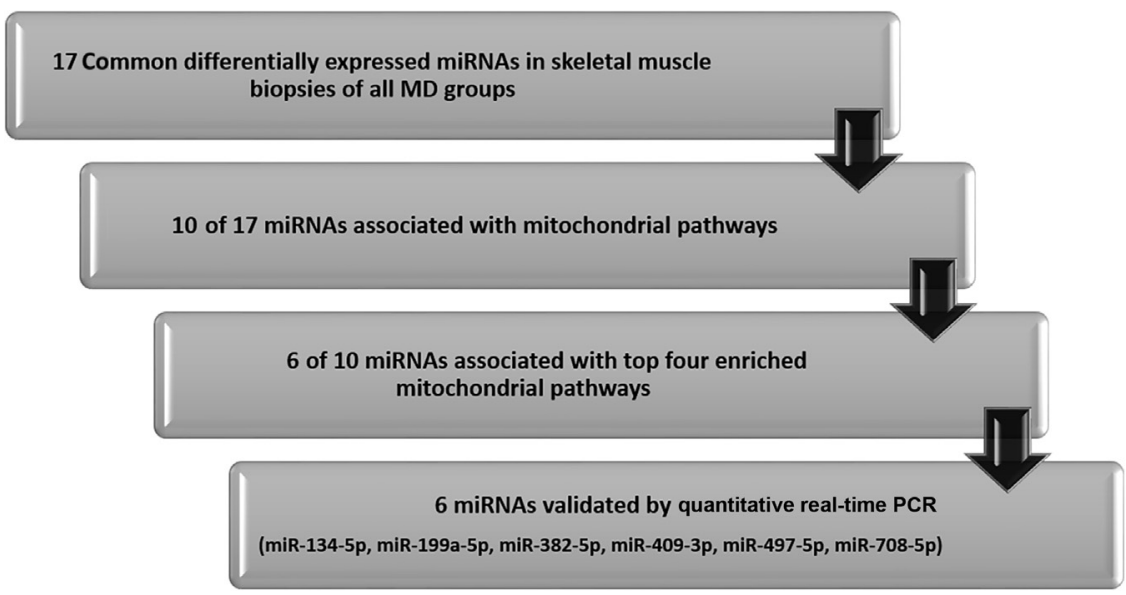

Figure 5 Flowchart representation of the pipeline for the identification of novel miRNAs associated with mitochondrial damage in muscular dystrophy (MD) groups. 
core myopathies, inflammatory myopathies/neuropathies, Charcot-Marie-Tooth neuropathy type 2, drug-induced peripheral neuropathies, spinal muscular atrophy, and amyotrophic lateral sclerosis. ${ }^{14}$ Among those diseases, mitochondrial dysfunction was detected mainly by ultra-high-resolution techniques. Two distinct studies analyzed skeletal muscles of Ullrich CMD patients by using electron microscopy and high-resolution fluorescence imaging, and they found swollen and perinuclear-localized mitochondria, disorganized cristae, and decreased membrane potential. ${ }^{10,31}$ In another study, Schiavone et $\mathrm{al}^{32}$ also detected decreased oxygen consumption rate in primary muscle cell cultures of DMD patients by extracellular flux analyzer, reduced mitochondrial membrane potential by using cationic fluorescent probes, and mitochondrial swelling by electron microscopy. Although the main reasons that cause secondary mitochondrial damage in skeletal muscles have not been identified yet, the potential role of small noncoding RNAs in these pathways is among the most prevalent research topics.

In this microarray-based study, we first identified 17 differentially expressed miRNAs in skeletal muscle tissues of all studied MD groups, 10 of which are directly related to mitochondrial pathways. Among these 10 miRNAs, 6 miRNAs, miR-134-5p, miR-199a-5p, miR-382-5p, miR-409-3p, miR-497-5p, and miR-708-5p, were selected as potential candidates as they are closely related to the top four enriched mitochondrial pathways (mitochondrial transport, organization, electron transport, and transmembrane transport) and were experimentally validated by quantitative real-time PCR analysis. Although the sample size is limited because they belonged to rare diseases that were representing the $12 \mathrm{pa}-$ tients, the strong relations of the differentially expressed miRNAs with the mitochondrial pathways related to neuromuscular phenotype may show the robustness of the microarray data obtained, even from limited number of samples. Also because of commonality approach, which is the main idea of our research, we aim to identify the common miRNA signatures underlying the pathogenesis of different muscular dystrophies. Two of these differentially expressed miRNAs (miR-134-5p and miR-382-5p) were previously shown to be associated with mitochondrial function in murine models. ${ }^{33,34}$ In a study including the miRNA microarray profiling of renal tissues in diabetic neuropathy mouse model, Qian et $\mathrm{al}^{34}$ (2018) observed that the up-regulation of miR134-5p further regulates the mitochondrial apoptosis process by targeting Bcl 2 mRNA in podocytes. In addition, silencing of the miR-382 was found to increase the mRNA levels of the genes PPARGC1A (PGC-1 $\alpha$ ), Sirtuin1 (SIRT1), Mitofusin 1 (MFN1), Mitofusin 2 (MFN2), and optic atrophy $1(O P A 1)$, and thus regulates mitochondrial biogenesis and dynamics. ${ }^{33}$ Although miR-708-5p, miR-497-5p, and miR199a-5p were shown to be important for the maintenance of skeletal muscle function, ${ }^{35-37}$ there are no other studies that describe the relationship between mitochondrial dysfunction and miR-409-3p, miR-497-5p, miR-708-5p, and miR-199a$5 \mathrm{p}$. However, compatible with the current study's findings, three of these differentially expressed miRNAs have been shown to dysregulate in other muscular dystrophies. miR497-5p was overexpressed in skeletal muscle of Pompe mouse model, as well as limb-girdle muscular dystrophy type $2 \mathrm{~A}$, limb-girdle muscular dystrophy type $2 \mathrm{~B}$, and facioscapulohumeral muscular dystrophy patients. ${ }^{38,39}$ Also, miR199a-5p was found to increase in skeletal muscle of DMD, limb-girdle muscular dystrophy type 2A, limb-girdle muscular dystrophy type 2B, and facioscapulohumeral muscular dystrophy patients. ${ }^{38}$ In addition, in a mouse model of Laminin $\alpha 2$ chain-deficient muscular dystrophy (dy3K/ dy3K), miR-409-3p was up-regulated. ${ }^{40}$ Although no study has been performed related with their functions, increasing of common miRNAs in other muscular dystrophies suggests their potential role in a common cellular pathway.

In conclusion, this is the first study in which we identified mitochondrial damage-associated common miRNA signatures in DMD, megaconial CMD, Ullrich CMD, and $\alpha$ DGpathy, each of which is caused by mutations in different nuclear genes encoding for proteins with distinct functions. The six elevated miRNAs that were identified as potential candidates might play a crucial role in common secondary mitochondrial pathomechanisms in skeletal muscle tissues. They have the potential to be common therapeutic targets for different MDs. miRNA-based therapies are among current research topics, and silencing of aberrantly expressed miRNAs by using chemically modified anti-miRNA oligonucleotides, miRNA sponges, or miRNA masking strategies has the potential to suppress the activity of these endogenous miRNAs and can be used for the prevention/reversal of mitochondrial damage observed as a common feature in different MD groups. ${ }^{41-45}$ By further exploring the effect of these common miRNAs on mitochondrial damage pathways and development of targeted therapeutic approaches will potentially help to better understand the pathogenesis of the diseases. Also, opportunity for identification of novel treatment options for many different diseases that are associated with mitochondrial damage may emerge. Our ongoing studies about in vitro functional analysis are being performed with these six common miRNAs to identify the most affected mitochondrial pathways and target genes of these miRNAs.

\section{Acknowledgment}

We thank the Hacettepe Teknokent Technology Transfer Center (HT-TTM) for manuscript editing services.

\section{Supplemental Data}

Supplemental material for this article can be found at http://doi.org/10.1016/j.ajpath.2020.06.011. 


\section{References}

1. Kaplan JC, Hamroun D: The 2016 version of the gene table of monogenic neuromuscular disorders (nuclear genome). Neuromuscul Disord 2015, 25:991-1020

2. Zatz M, Passos-Bueno MR, Vainzof M: Neuromuscular disorders: genes, genetic counseling and therapeutic trials. Genet Mol Biol 2016, 39:339-348

3. Durbeej M, Campbell KP: Muscular dystrophies involving the dystrophin-glycoprotein complex: an overview of current mouse models. Curr Opin Genet Dev 2002, 12:349-361

4. Castro-Gago M, Dacruz-Alvarez D, Pintos-Martínez E, BeirasIglesias A, Arenas J, Martín MÁ, Martínez-Azorín F: Congenital neurogenic muscular atrophy in megaconial myopathy due to a mutation in CHKB gene. Brain Dev 2016, 38:167-172

5. Gutierrez Rios P, Kalra AA, Wilson JD, Tanji K, Akman HO, Area Gomez E, Schon EA, DiMauro S: Congenital megaconial myopathy due to a novel defect in the choline kinase Beta gene. Arch Neurol 2012, 69:657-661

6. Irwin WA, Bergamin N, Sabatelli P, Reggiani C, Megighian A, Merlini L, Braghetta P, Columbaro M, Volpin D, Bressan GM, Bernardi P, Bonaldo P: Mitochondrial dysfunction and apoptosis in myopathic mice with collagen VI deficiency. Nat Genet 2003, 35:367-371

7. Mitsuhashi S, Hatakeyama H, Karahashi M, Koumura T, Nonaka I, Hayashi YK, Noguchi S, Sher RB, Nakagawa Y, Manfredi G, Goto Y-I, Cox GA, Nishino I: Muscle choline kinase beta defect causes mitochondrial dysfunction and increased mitophagy. Hum Mol Genet 2011, 20:3841-3851

8. Rybalka E, Timpani CA, Cooke MB, Williams AD, Hayes A: Defects in mitochondrial ATP synthesis in dystrophin-deficient Mdx skeletal muscles may be caused by complex I insufficiency. PLoS One 2014, 9:e115763

9. Shkryl VM, Martins AS, Ullrich ND, Nowycky MC, Niggli E, Shirokova N: Reciprocal amplification of ROS and Ca2 + signals in stressed mdx dystrophic skeletal muscle fibers. Pflügers Archiv 2009, 458:915-928

10. Tagliavini F, Sardone F, Squarzoni S, Maraldi NM, Merlini L, Faldini C, Sabatelli P: Ultrastructural changes in muscle cells of patients with collagen VI-related myopathies. Muscles Ligaments Tendons J 2013, 3:281-286

11. Vila MC, Rayavarapu S, Hogarth MW, Van der Meulen JH, Horn A, Defour A, Takeda Si, Brown KJ, Hathout Y, Nagaraju K, Jaiswal JK: Mitochondria mediate cell membrane repair and contribute to Duchenne muscular dystrophy. Cell Death Differ 2017, 24:330-342

12. Aksu E, Talim B, Balci-Hayta B: Impaired Balance of Mitochondrial Fission and Fusion in an Invitro Model of Mitochondrial Unfolded Protein Response. Copenhagen, Denmark, The European Human Genetics Conference, 2017

13. Aksu E, Talim B, Dincer P, Balci-Hayta B: Activation of the Mitochondrial Unfolded Protein Response (UPRmt) Pathway in C2C12 Myoblast Cell Line, The European Human Genetics Conference. Eur J Hum Genet 2016, 24 E-Supp:221-222

14. Katsetos CD, Koutzaki S, Melvin JJ: Mitochondrial dysfunction in neuromuscular disorders. Semin Pediatr Neurol 2013, 20:202-215

15. Merlini L, Nishino I: 201st ENMC International Workshop: autophagy in muscular dystrophies-translational approach, 1-3 November 2013, Bussum, The Netherlands. Neuromuscul Disord 2014, 24:546-561

16. Romanello V, Guadagnin E, Gomes L, Roder I, Sandri C, Petersen Y, Milan G, Masiero E, Del Piccolo P, Foretz M, Scorrano L, Rudolf R, Sandri M: Mitochondrial fission and remodelling contributes to muscle atrophy. EMBO J 2010, 29:1774-1785

17. Wu L, Fan J, Belasco JG: MicroRNAs direct rapid deadenylation of mRNA. Proc Natl Acad Sci U S A 2006, 103:4034-4039

18. Duarte FV, Palmeira CM, Rolo AP: The role of microRNAs in mitochondria: small players acting wide. Genes (Basel) 2014, 5: 865-886
19. Li P, Jiao J, Gao G, Prabhakar BS: Control of mitochondrial activity by miRNAs. J Cell Biochem 2012, 113:1104-1110

20. Etheridge A, Lee I, Hood L, Galas D, Wang K: Extracellular microRNA: a new source of biomarkers. Mutat Res 2011, 717:85-90

21. Zaharieva IT, Calissano M, Scoto M, Preston M, Cirak S, Feng L, Collins J, Kole R, Guglieri M, Straub V, Bushby K, Ferlini A, Morgan JE, Muntoni F: Dystromirs as serum biomarkers for monitoring the disease severity in Duchenne muscular dystrophy. PLoS One 2013, 8:e80263

22. Carrasco-Rozas A, Fernandez-Simon E, Lleixa MC, Belmonte I, Pedrosa-Hernandez I, Montiel-Morillo E, Nunez-Peralta C, Llauger Rossello J, Segovia S, De Luna N, Suarez-Calvet X, Illa I, DiazManera J, Gallardo E: Identification of serum microRNAs as potential biomarkers in Pompe disease. Ann Clin Transl Neurol 2019, 6: $1214-1224$

23. Yamamoto H, Morino $\mathrm{K}$, Nishio $\mathrm{Y}$, Ugi S, Yoshizaki $\mathrm{T}$, Kashiwagi A, Maegawa H: MicroRNA-494 regulates mitochondrial biogenesis in skeletal muscle through mitochondrial transcription factor A and Forkhead box j3. Am J Physiol Endocrinol Metab 2012, 303:E1419-E1427

24. Goljanek-Whysall K, Soriano-Arroquia A, McCormick R, Chinda C, McDonagh B: miR-181a regulates p62/SQSTM1, Parkin and protein DJ-1 promoting mitochondrial dynamics in skeletal muscle ageing. bioRxiv 2019:805176

25. Mohamed JS, Hajira A, Pardo PS, Boriek AM: MicroRNA-149 inhibits PARP-2 and promotes mitochondrial biogenesis via SIRT1/PGC-1 $\alpha$ network in skeletal muscle. Diabetes 2014, 63:1546-1559

26. Garros RF, Paul R, Connolly M, Lewis A, Garfield BE, Natanek SA, Bloch S, Mouly V, Griffiths MJ, Polkey MI, Kemp PR: MicroRNA542 promotes mitochondrial dysfunction and SMAD activity and is elevated in intensive care unit-acquired weakness. Am J Respir Crit Care Med 2017, 196:1422-1433

27. Boycott KM, Lau LP, Cutillo CM, Austin CP: International collaborative actions and transparency to understand, diagnose, and develop therapies for rare diseases. EMBO Mol Med 2019, 11:e10486

28. Roy AJ, Van den Bergh P, Van Damme P, Doggen K, Van Casteren V: The BSC: early stages of building a rare disease registry, methods and 2010 data from the Belgian Neuromuscular Disease Registry (BNMDR). Acta Neurol Belg 2015, 115:97-104

29. Hoy SM: Nusinersen: first global approval. Drugs 2017, 77:473-479

30. McDonald CM, Campbell C, Torricelli RE, Finkel RS, Flanigan KM, Goemans N, Heydemann P, Kaminska A, Kirschner J, Muntoni F, Osorio AN, Schara U, Sejersen T, Shieh PB, Sweeney HL, Topaloglu H, Tulinius M, Vilchez JJ, Voit T, Wong B, Elfring G, Kroger H, Luo X, McIntosh J, Ong T, Riebling P, Souza M, Spiegel RJ, Peltz SW, Mercuri E: Ataluren in patients with nonsense mutation Duchenne muscular dystrophy (ACT DMD): a multicentre, randomised, doubleblind, placebo-controlled, phase 3 trial. Lancet 2017, 390:1489-1498

31. Angelin A, Tiepolo T, Sabatelli P, Grumati P, Bergamin N, Golfieri C, Mattioli E, Gualandi F, Ferlini A, Merlini L, Maraldi NM, Bonaldo P, Bernardi P: Mitochondrial dysfunction in the pathogenesis of Ullrich congenital muscular dystrophy and prospective therapy with cyclosporins. Proc Natl Acad Sci U S A 2007, 104:991-996

32. Schiavone M, Zulian A, Menazza S, Petronilli V, Argenton F, Merlini L, Sabatelli P, Bernardi P: Alisporivir rescues defective mitochondrial respiration in Duchenne muscular dystrophy. Pharmacol Res 2017, 125:122-131

33. Dahlmans D, Houzelle A, Andreux P, Wang X, Jorgensen JA, Moullan N, Daemen S, Kersten S, Auwerx J, Hoeks J: MicroRNA382 silencing induces a mitonuclear protein imbalance and activates the mitochondrial unfolded protein response in muscle cells. J Cell Physiol 2019, 234:6601-6610

34. Qian X, Tan J, Liu L, Chen S, You N, Yong H, Pan M, You Q, Ding D, Lu Y: MicroRNA-134-5p promotes high glucose-induced podocyte apoptosis by targeting bcl-2. Am J Transl Res 2018, 10:989-997

35. Alexander MS, Kawahara G, Motohashi N, Casar JC, Eisenberg I, Myers JA, Gasperini MJ, Estrella EA, Kho AT, Mitsuhashi S, 
Shapiro F, Kang PB, Kunkel LM: MicroRNA-199a is induced in dystrophic muscle and affects WNT signaling, cell proliferation, and myogenic differentiation. Cell Death Differ 2013, 20: $1194-1208$

36. Fiorillo AA, Tully CB, Damsker JM, Nagaraju K, Hoffman EP, Heier CR: Muscle miRNAome shows suppression of chronic inflammatory miRNAs with both prednisone and vamorolone. Physiol Genomics 2018, 50:735-745

37. Guilbaud M, Gentil C, Peccate C, Gargaun E, Holtzmann I, Gruszczynski C, Falcone S, Mamchaoui K, Ben Yaou R, Leturcq F, Jeanson-Leh L, Pietri-Rouxel F: miR-708-5p and miR-34c-5p are involved in nNOS regulation in dystrophic context. Skelet Muscle 2018, 8:15

38. Eisenberg I, Eran A, Nishino I, Moggio M, Lamperti C, Amato AA, Lidov HG, Kang PB, North KN, Mitrani-Rosenbaum S, Flanigan KM, Neely LA, Whitney D, Beggs AH, Kohane IS, Kunkel LM: Distinctive patterns of microRNA expression in primary muscular disorders. Proc Natl Acad Sci U S A 2007, 104:17016-17021

39. Tarallo A, Carissimo A, Gatto F, Nusco E, Toscano A, Musumeci O, Coletta M, Karali M, Acampora E, Damiano C, Minopoli N, Fecarotta S, Della Casa R, Mongini T, Vercelli L, Santoro L, Ruggiero L, Deodato F, Taurisano R, Bembi B, Dardis A, Banfi S,
Pijnappel WWP, van der Ploeg AT, Parenti G: microRNAs as biomarkers in Pompe disease. Genet Med 2019, 21:591-600

40. Moreira Soares Oliveira B: Potential Therapies and Biomarkers for LAMA2-CMD: Does the microRNA Hype Deliver? Lund, Sweden, Lund University, 2018. pp. 54

41. Czauderna F, Fechtner M, Dames S, Aygun H, Klippel A, Pronk GJ, Giese K, Kaufmann J: Structural variations and stabilising modifications of synthetic siRNAs in mammalian cells. Nucleic Acids Res 2003, 31:2705-2716

42. Lavenniah A, Luu TDA, Li YP, Lim TB, Jiang J, Ackers-Johnson M, Foo RS: Engineered circular RNA sponges act as miRNA inhibitors to attenuate pressure overload-induced cardiac hypertrophy. Mol Ther 2020, 28:1506-1517

43. Lee JB, Hong J, Bonner DK, Poon Z, Hammond PT: Self-assembled RNA interference microsponges for efficient siRNA delivery. Nat Mater 2012, 11:316-322

44. Lennox KA, Behlke MA: A direct comparison of anti-microRNA oligonucleotide potency. Pharm Res 2010, 27:1788-1799

45. Weiler J, Hunziker J, Hall J: Anti-miRNA oligonucleotides (AMOs): ammunition to target miRNAs implicated in human disease? Gene Ther 2006, 13:496-502 